European Journal of Social Psychology

Eur. J. Soc. Psychol. 30, 799-811 (2000)

\title{
Assimilation and contrast in social comparisons as a consequence of self- construal activation
}

\author{
ULRICH KÜHNEN $^{1}$ and BETTINA HANNOVER ${ }^{2 *}$ \\ ${ }^{1}$ University of Michigan, USA \\ ${ }^{2}$ University of Dortmund, Germany
}

\begin{abstract}
We extended existing research about self-construal activation to the study of social comparisons, specifically to self-other similarity ratings. Independent self-knowledge substantiates the notion of dissimilarity, whereas interdependent self-knowledge implies similarity with others. Therefore, perceived self-other similarity was predicted to decrease after independent and increase after interdependent self-knowledge priming. However, we expected such assimilation effects to occur only if the priming was subtle, but contrast effects if it was overt. In order to test this hypothesis, we developed a scrambled sentences test for priming the respective self-knowledge. The unscrambled sentences described the self either in terms of independence or interdependence. The subtlety of this priming was manipulated by having participants write down either the full sentences (overt priming) or only the remaining word in each item (subtle priming). Results confirmed the predictions. Underlying cognitive processes of the effects are discussed. Copyright (C) 2000 John Wiley Sons, Ltd.
\end{abstract}

\section{INTRODUCTION}

Considerable attention has been paid to the differentiation between interdependent and independent self-construals. In particular, it has been suggested that the way in which people construe their selves influences the very nature of personal experiences, behavior and cognition. The present research was conducted with two goals. First, to

* Correspondence to: Prof. Dr Bettina Hannover, Universität Dortmund, Fachbereich 14, Emil-Figg-Str. 50,44221 Dortmund, Germany. e-mail: hannover@wap-mail.fb14.uni-dortmund.de

Contract/grant sponsor: Deutsche Forschungsgemeinschaft.

Contract/grant number: HA2381/3-1. 
extend existing research on self-construal priming to social comparisons we speculate that independent self-knowledge substantiates the notion of being dissimilar from others, whereas interdependent self-cognitions give evidence for being similar to others. Accordingly, we expected the activation of interdependent or independent self-construals to influence self-other similarity ratings. Second, we applied social cognition findings about assimilation and contrast following semantic priming to the study of self-construal activation. We examined whether or not the direction of the priming influence was a function of its subtlety. Given subtle priming, we expected self-other similarity ratings to be assimilated toward the implications of the activated self-knowledge, while contrasted similarity ratings were expected following overt selfconstrual priming.

\section{INTER-INDIVIDUAL DIFFERENCES IN THE RELATIVE ACCESSIBILITY OF INTERDEPENDENT AND INDEPENDENT SELF-CONSTRUALS}

Markus and Kitayama (1991) regard culture as a set of often implicit normative tasks one has to fulfill in order to be a good person. In individualist societies, the cultural imperative to be a unique and independent person and to express this openly prevails (e.g. Shweder \& Bourne, 1984). As a consequence, the self is predominantly defined in terms of internal unique features such as traits, abilities, and attitudes (e.g. Oyserman \& Markus, 1996; Trafimow, Triandis \& Goto, 1991; Rhee, Uleman, Lee \& Roman, 1995). The collectivist cultural imperative, however, is to be a dependable member of relevant social groups. Therefore, from a collectivist perspective the self is perceived as fundamentally related to others. Identity is defined primarily by a person's relationships with others, group memberships or social roles (Markus, Kitayama \& Heiman, 1997; Oyserman \& Markus, 1996; Trafimow et al., 1991).

In an attempt to solve the culturally pre-determined developmental tasks (Markus \& Kitayama, 1991), individuals acquire self-construals that are related to these tasks. Over time these construals become chronically highly accessible (Higgings \& King, 1981). Culture may thus be understood as a chronic source of activation of different self-construals (Hannover, 1997). Social cognition research has provided substantial empirical evidence that accessible constructs shape our view of others and of ourselves.

In applying these considerations to social comparison judgments, one may predict different degrees of perceived similarity of the self to others between the cultures. Within the independent perspective the self is primarily defined in terms of internal attributes. Therefore, independent self-knowledge substantiates the notion of uniqueness of the self. On the other hand, higher perceived self-other similarity can be expected for participants with an interdependent self-construal, because interdependent features are shared with others. Cross-cultural differences in perceived self-other similarity ratings between individualist and collectivist cultures (see Kühnen, 1999, for a review) support this prediction. For instance, when comparing themselves and others with respect to personal skills, Goethals, Messick and Allison (1991) found that people in individualist societies underestimate the percentage of others who do as well (or even better) than they do. Markus and Kitayama (1991) found this tendency to be attenuated in collectivist culture members. Similarily, when 
directly asked to assess the similarity to others, Kühnen (1999) found people with chronically accessible independent self-knowledge to produce lower ratings than people with chronically accessible interdependent self-construals. To summarize, independent self-knowledge implies dissimilarity from others, whereas interdependent self-knowledge implies similarity with them.

\section{PRIMING INDEPENDENT VERSUS INTERDEPENDENT SELF- CONSTRUALS}

The effects of independent and interdependent self-construals on social comparison judgments cannot directly be tested within the quasi-experimental designs typically used in cross-cultural studies. This test is only possible through the experimental manipulation of the respective self construals' accessibility. If cultural differences can be accounted for by the chronic accessibility of the respective self-construals, experimentally priming them should mirror such inter-individual differences.

Following this reasoning, Trafimow et al. (1991) randomly assigned research participants from individualist (USA) or collectivist cultures (Chinese students, who lived in the USA) to one of two priming conditions. In the condition designed to activate private (i.e. independent) self-construals, participants were asked to think about differences between themselves and their family and friends. In the other condition aimed at activating collective (i.e. interdependent) self-construals, research participants were asked to think about what they had in common with their family and friends. Subsequently, Trafimow et al. administered the Twenty Statements Test (TST; Kuhn \& McPartland, 1954), i.e. participants had to respond to 20 'Who am I?' probes. The authors found that participants who had previously thought about differences described themselves more frequently using independent self-construals (e.g. 'I am intelligent') than interdependent ones (e.g. 'I am Tom's sister'). This difference was reversed in participants who had previously thought about their similarities with others. The same pattern of results was observed when comparing participants from individualist with participants from collectivist cultural backgrounds. Thus, differences in self-descriptions of individualist and collectivist culture members were mirrored in the two priming conditions.

Similar results were obtained from another priming task. In this experiment, participants read a story about an ancient warrior 'Tiglath' who was put in command of the troops for a difficult mission by his King 'Sostoras'. The authors manipulated the explanation of why King Sostoras chose Tiglath. In the private self-priming condition (i.e. independent priming), Tiglath was described as a 'talented general', whereas in the collective self-priming condition (i.e. interdependent priming) participants learned that Sostoras chose Tiglath because he 'was a member of his family' (Trafimow et al., 1991, p. 112).

In a more recent study, Trafimow, Silverman, Mei-Tai Fan \& Shui Fan Law (1997) replicated these results with Chinese participants who live in their native country. Trafimow and Smith (1998) extended this research to the study of Native Americans. They replicated the results one more time, hence supporting the general notion that priming private (i.e. independent) or collective (i.e. interdependent) self-construals affects subsequent self-descriptions. Together, the studies by Trafimow and his 
colleagues give experimental evidence that people describe themselves in terms of highly accessible self-knowledge.

Recently, Gardner, Gabriel \& Lee (1999) extended the research on self-construal priming to studying attitudes and social values. They used a priming method first introduced by Brewer and Gardner (1996). These authors had their research participants read a brief descriptive paragraph about a trip to a city and circle all pronouns that appeared in the text. In one condition, almost all of the pronouns were independent ones (like 'I', 'mine', etc.), while in the other condition interdependent pronouns ('we', 'our', etc.) prevailed. Following the priming task, research participants were asked to complete a values inventory (Schwartz, 1992), a social judgment task which aspired to measure the extent to which interpersonal norms of helping behavior were seen as objective obligations (Miller, Bersoff \& Harwood, 1990) and, finally, the Twenty Statements Test (Kuhn \& McPartland, 1954). Gardner et al. (1999) found that the differences between the two priming conditions reflected the differences between individualist and collectivist culture members. Participants in the independent priming condition endorsed more individualist social values, perceived social obligations as less important, and described the self more in terms of independent self-construals than participants in the interdependent priming condition (Experiment 1). In a second experiment, Gardner et al. replicated their results with participants having either an individualist cultural background (USA) or a more collectivist one (Hong Kong).

Self-descriptions and the importance of personal values are not the only variables shown to be affected by the accessibility of independent or interdependent selfconstruals. Further research has investigated consequences of self-construal priming for the relative importance of subjective norms and attitudes when predicting behavioral intentions. As Trafimow and Finlay (1996) have shown, the stronger a person's collective (i.e. interdependent) self, the more he or she intends to behave in accordance with subjective norms rather than with personal attitudes. Ybarra and Trafimow (1998) obtained similar results, when using a priming technique to either activate private (i.e. independent) self-construals or collective (i.e. interdependent) ones.

In summary, the above-mentioned studies substantiate the notion that selfdescriptions, the importance of personal values, and behavioral intentions are influenced by the accessibility of either independent or interdependent self-construals. Additionally, these studies demonstrate that cultural differences in self-construals can be simulated by experimental priming. This in turn suggests that culture affects human cognition and behavior via the relative accessibility of independent or interdependent self-construals.

\section{THE INFLUENCE OF SELF-CONSTRUALS ON SOCIAL COMPARISON PROCESSES}

In this paper, we want to extend the existing research to social comparison judgments. Specifically, we investigated how priming of independent or interdependent selfconstruals affects self-other similarity ratings. How similar a person regards another person to the self is a crucial factor for the comparisons' outcome (Mussweiler, 2000). 
In turn these outcomes influence our individual experiences in numerous ways (see Mussweiler \& Strack, in press, for a recent review).

In our study, we expected that self-other similarity ratings would be assimilated toward the implications of independent and interdependent self-knowledge. Specifically, since we assumed that independent self-knowledge substantiates dissimilarity to others, priming self-cognitions of this kind should decrease the perceived self-other similarity. Interdependent self-construals, however, describe features one has in common with others. Activating these self-aspects should consequently increase the perceived similarity with others.

\section{SUBTLETY OF THE PRIMING AS A PREDICTOR OF THE DIRECTION OF THE INFLUENCE}

So far we have only considered that judgments are assimilated toward the implications of activated mental categories. As previous research has shown, however, this is not always the case. If a contextual priming influence is suspected, participants may engage in correctional attempts, in order to level out the priming effect. For instance, in a study by Strack, Schwarz, Bless, Kübler \& Wänke (1993) participants were asked to judge a target person based on a brief description of his behavior, which could be interpreted as evidence for either positive or negative traits. In an ostensibly unrelated previous task the relevant trait categories had been primed. In order to elicit the participants' awareness of this influence half of them were reminded of the priming episode right before they were to judge the ambiguous target person. The judgments of those participants, whose attention had not been directed to the priming influence, were assimilated toward the activated trait categories. Those who had been reminded of the priming showed the opposite pattern. Their judgments were more positive, if a negative trait had been primed and vice versa. The authors interpret this contrast effect as a result of correction processes. Since the participants in the overt priming condition have presumably recognized the 'irrelevant' influence that contributed to their interpretation of the target person, they may have corrected their judgments in the direction opposite to the priming influence, resulting in a contrast effect (see Strack \& Hannover, 1996, for a more detailed discussion).

Moskowitz and Roman (1992) tested the hypothesis that the subtlety of the priming predicts the direction of its influence by using a less blatant experimental procedure. They administered a series of trait implying sentences describing a target person's behavior. By varying the research participants' judgment task, the authors manipulated the subtlety of the priming. In one condition participants were asked to simply memorize the sentences (i.e. subtle priming), whereas in the overt priming condition, they were asked to form an impression of the described target person, thus becoming aware of the primed trait concepts. In a subsequent judgment task about a different target person, participants in the subtle priming condition showed judgments that were assimilated toward the primed trait concepts. The opposite effect appeared in the overt priming condition.

Based on these findings, it can be stated that priming can induce either assimilation or contrast effects. If the priming is subtle, subsequent judgments will probably be 
assimilated toward the implications of the activated categories. If the priming is overt, however, contrasted judgments are more likely to be observed.

In this study, we applied these considerations to the particular case of self-related judgments. We have reviewed those studies in which either independent or interdependent self-construals were primed. In all cited studies, the priming was subtle. Accordingly, assimilation effects were found. In this study, we experimentally manipulated the subtlety of the priming. If the priming is subtle, subsequent selfother similarity ratings should be assimilated toward the content of the activated selfknowledge (i.e. participants in the independent priming condition should perceive a smaller similarity with others than participants in the interdependent priming condition). If, however, the priming influence is overt, subsequent judgments are more likely to be the opposite of this influence. As a consequence, participants with independent priming are expected to rate themselves as being more similar to others than participants in the interdependent priming condition.

\section{METHOD}

\section{Participants}

One hundred students from the University of Dortmund (Germany) were recruited as part of a course requirement in an introductory seminar of social psychology. They were randomly assigned to the experimental conditions described below.

\section{Design}

The content of the primed self-construals (independent versus interdependent) and the subtlety of the priming (subtle versus overt) were varied between subjects. Since the experiment was introduced as consisting of two independent studies, we also varied the order of instructions as a control factor. Half of the participants received the instructions for both tasks in the beginning. The other half was given the instruction for the second task (i.e. the dependent variable) immediately before they worked on it (i.e. after they had been primed). Thus, the experiment consisted of 2 (primed concepts) $\times 2$ (subtlety of the priming) $\times 2$ (order of instruction) factorial design.

\section{Material}

In order to activate independent or interdependent self-construals we constructed a scrambled sentences test (Srull \& Wyer, 1979) in which participants receive a series of five words in a randomized order. The participants' task is to create a meaningful and grammatically correct sentence with four out of the five words. As a result, their attention is drawn to the syntax of the sentences, rather than to the semantic meaning. Consequently, different cognitive categories can be activated without the participants' awareness of the influence, by simply varying the content of the complete sentences (Srull \& Wyer, 1979).

Our scrambled sentences test consisted of 24 relevant items (i.e. either independent or interdependent self-cognitions) and three filler items. 


\section{Priming Independent Versus Interdependent Self-cognitions}

Every item consisted of five words, four of which were to be used to build a grammatically correct sentence. The resulting sentences were self-descriptions. Because of the particular verb conjugations used in the material, for each item only one solution was possible. ${ }^{1}$ In one condition, all 24 relevant items resulted in independent self-descriptions. Additionally, the fifth, superfluous word of each item also implied some form of independence. For example 'unique like being dissociate I' results in the independent self-description 'I like being unique'. Other examples are 'impressionable I not am assertiveness' or 'I ideas have unusual autonomy'. In the second priming condition the resulting sentences described interdependent aspects of the self. Again, the unnecessary fifth words were also associated with interdependence. For instance 'help like group I to' results in 'I like to help'. Other examples are 'respect partnership others' I opinions' or 'I together team my support'. As mentioned before, three out of twenty-seven items were neutral to the primed concepts. These filler items read 'reading life like books I', 'forgettable tasks avoid boring I', and 'really love I nice nature'.

\section{Subtlety of the Priming}

Scrambled sentences are generally used to activate cognitive concepts without the research participants realizing it. However, our intention was to show that the direction of the influence depends on the subtlety of the priming. This was achieved by varying the salience of the self-descriptions. In the subtle priming condition the participants' task was to isolate and write down the remaining word of each scrambled sentence. We assumed that while working on this task, participants complete the sentences without reflecting upon their validity as self-descriptions. As a consequence, subsequent similarity judgments should be assimilated toward the activated self-knowledge, i.e. participants in the independent priming condition were expected to judge themselves as less similar to others than participants in the interdependent priming condition.

In order to render the priming influence more salient in the overt priming condition, participants were asked to write down the complete unscrambled sentences. We assumed that writing down 24 sentences that describe the self as being unique, independent, self-confident, etc., or as helpful, interdependent, supportive, etc., is a blatant and easily identifiable influence. Participants in this overt priming group were expected to contrast their judgements with the primed selfconstruals. Consequently, participants in the independent priming condition were expected to judge themselves as more similar to others than participants in the interdependent priming condition.

\section{Procedure}

All participants were given a booklet to fill out. The front page was the same for all participants. It said that the booklet contained two independent studies, one concerning language processing and the other concerning social judgments.

\footnotetext{
${ }^{1}$ Note that since the items are presented in English language here, there is more than one solution possible for each item. This was not the case in the German version we used.
} 
The first task, the priming, was introduced as a language test. Instructions for the scrambled sentences task was explained on a cover page through an example sentence which was non-related to independence-interdependence. In the subtle priming condition the participants' task was to build the possible four-word sentence out of every set of five randomly presented words, but to write down only the remaining fifth word of each item. In the overt priming condition participants were instructed to write down the completed sentences. On the next page, the 27 scrambled sentences were presented, with a graphical answering scale as described below next to each item. The third page of the questionnaire gave the instructions for the ostensibly unrelated second task, which was introduced as concerned with 'how we make similarity judgments about ourselves and others'. Participants were asked to think of another student of the same sex, who was neither a close friend, nor completely unknown. The following five similarity questions were asked:

(1) How similar is the other person to you in a group discussion?

(2) How similar is the other person to you at a party?

(3) How similar is the other person to you in a work-group with other students?

(4) How similar is the other person to you during an evening out with your friends?

(5) How similar is the other person to you when talking about personal problems with other students?

We chose these questions because they are relatively neutral in regard to the emotional consequences of the comparisons' outcomes. A graphical rating scale was used for measuring the perceived self-other similarity. Participants were given a line between the poles 'very dissimilar' and 'very similar' and were asked to mark their perceived similarity with a cross. The dependent variable (i.e. judged similarity) was measured as the distance in millimeters of the participants' cross from the left pole representing dissimilarity on each answering scale. Since the maximal distance was 10 centimeters, the answers could vary between 0 (representing 'very dissimilar') and 100 (indicating 'very similar').

As mentioned above, the order of instructions was varied as a control factor. Only half the participants received the instructions in the above described order. The other half received the instructions for both studies at the beginning.

\section{RESULTS}

The five similarity ratings were averaged for each participant. The reliability of the five ratings reached an alpha of 0.67 . In order to test our hypothesis mean similarity ratings were subjected to a 2 (primed self-knowledge) $\times 2$ (subtlety of priming) $\times 2$ (order of instructions)-factorial analysis of variance. Since the order of instructions did not produce any significant effects, it was excluded from any further analysis. As expected, the $2 \times 2$-factorial ANOVA revealed a reliable interaction between the two experimental factors, $F(1,95)=4.48 ; p=0.037$. In the subtle condition (where participants were asked to determine and write down the superfluous word), priming independent self-knowledge led to smaller judged similarity $(M=42.82$; $S D=15.02)$ than priming interdependent self-knowledge $(M=48.9 ; S D=13.39)$, $t(48)=1.51 ; p=0.07$. Apparently similarity ratings had been assimilated toward the 
semantic content of the activated concepts as predicted for this condition. In the overt priming condition (where participants had to write down the complete sentences) the opposite effect was observed. As expected, participants in the independent priming condition judged themselves as less similar to others $(M=54 ; S D=16.67)$ than participants in the interdependent priming condition $(M=46,9 ; S D=16.52)$, $t(48)=1.49 ; p=0.07$.

\section{DISCUSSION}

This study had two main goals. First, we wanted to extend the literature on consequences of self-construal activation to the study of social comparisons. Interdependent self-cognitions describe characteristics one shares with others, hence they support the notion of self-other similarity. Independent self-construals on the other hand, describe unique personal features substantiating self-other dissimilarity. Therefore, we expected the activation of independent self-knowledge to decrease the assumed similarity between the self and others, but priming interdependent self-knowledge to result in increased similarity ratings. Our second goal was to provide the first experimental evidence that priming independent versus interdependent self-knowledge does not always lead to assimilation in subsequent judgments. Rather, we expected the direction of the priming to be a function of its subtlety. If the priming was subtle, subsequent self-other similarity ratings were expected to be assimilated to the activated self-knowledge, while the reverse pattern was predicted if the priming influence was overt.

Despite the fact that comparing the relevant conditions revealed only marginally significant differences, the interaction effect was significant and the mean pattern was in line with our predictions. The priming influence was assumed to be subtle if the participants were asked to write down only the remaining word of each scrambled sentence. Given subtle priming, participants rated themselves less similar to others in the independent than in the interdependent priming condition. Thus, the direction of the priming influence reflected the implications of activated self-knowledge (i.e. an assimilation effect could be observed). When participants had to write down the complete unscrambled sentences during the priming phase, subsequent similarity ratings showed the opposite pattern.

In our study we affirm the relevance of the priming of independent versus interdependent self-knowledge for social judgments. With the scrambled sentences test developed for the present study, we used a priming technique that differs from those employed in previous studies (Gardner et al., 1999; Trafimow et al., 1991). The effects of self-construal activation were reliably found across these different types of studies, testifying to the robustness and generality of the findings. More importantly, however, the present findings open a new research field for studying self-construal activation, namely social comparisons. The degree of perceived similarity to others is a crucial mediator in determining the consequences of social comparisons (Mussweiler, 2000). In this study we used dimensions of comparison which are relatively neutral in regards to the evaluative implications of the comparison's outcomes: It is not obvious whether perceiving the self as either similar or dissimilar to others 'at a party' is beneficial for a persons' self-esteem. It can be assumed that the 
effects of self-construal activation will be even stronger if the consequences of perceived similarity to others have clear-cut consequences for self-evaluations. Further research using different dimensions of comparison is needed to test these assumptions.

To our knowledge, this study is the first to test the hypothesis that self-construal activation does not always result in the assimilation of subsequent judgments. Priming self-construals in an overt versus covert manner resulted in opposite effects. Based on previous findings (Moskowitz \& Roman, 1992; Strack et al., 1993) we expected and found assimilation effects only if self-knowledge had been activated in a subtle manner. If the priming was overt, however, the subsequent similarity ratings revealed an opposite effect. It is conceivable that contrast effects not only become evident in social comparison judgments, but also in self-descriptions, behavioral intentions, and personal values. In these contexts, self-construal activation has already been shown to result in assimilation (Gardner et al., 1999; Trafimow \& Finlay, 1996; Ybarra \& Trafimow, 1998).

Although our results are in line with the predictions, they remain ambiguous with respect to the exact judgmental mechanism underlying the observed contrast effect in the overt priming condition. Referring to the work by Strack et al. (1993) and by Moskowitz and Roman (1992), we have suggested that the contrast effect observed in the overt priming condition is due to our participants attempting to correct the influence of the priming on their judgments. In particular, writing down the full unscrambled sentences should have directed the participants' attention to the content of the self-descriptions. In this situation, it is conceivable that participants believed the priming task influenced their similarity-judgments and they may have tried to counteract this influence (Moskowitz \& Roman, 1992). More precisely, participants may have reflected about the validity of the created sentences. In the majority of cases, individuals consider themselves both interdependent and independent (Niedenthal \& Beike, 1997). Therefore, writing down sentences that describe the self as exclusively independent or exclusively interdependent, may be experienced as unrepresentative for the self. This can induce participants to correct their judgments by selectively accessing inconsistent knowledge or by adapting their response on the provided answering scale (Strack, 1992). In either case, the contrast effects observed in the overt priming condition can be explained as correction contrast, i.e. from participants being aware of a potential influence and correcting their similarity ratings by moving them away from their reactions to the priming.

It could be argued, however, that correction processes do not necessarily result in contrast, but instead may lead to assimilation. In their flexible correction model, Wegener and Petty (1997) suggest that corrections correspond to respondents' naive theories of how a potentially biasing factor may influence their judgments. If people assume the uncorrected effect of a context to be assimilation, they may correct their judgments by adjusting their ratings from the context (correction contrast). However, if they view some stimulus as likely to produce contrast, they may as well adjust their judgments toward the context (correction assimilation). Applied to our findings, one may wonder if both the contrast effect in the overt priming condition and the assimilation effect in the subtle priming condition can be traced back to theory-based correction. This does not seem plausible, because it would require participants to develop 
opposite subjective theories about the direction of the influence if the priming was overt or subtle. It makes more sense to assume that our participants used theory-based correction only in the overt priming condition. A prerequisite for theory-based correction is that the person becomes aware of a potential bias. Therefore, it seems unlikely that the assimilation effect observed in our subtle priming condition is due to theory-based correction. In this way, our interpretation that there was correction contrast in the overt priming condition only becomes compatible with the one suggested by Wegener and Petty's (1997) flexible correction model.

An opposing interpretation of our data is the possibility of comparative contrast. Herr (1986) has shown that, while priming trait categories (e.g. hostility) results in assimilation effects, priming extreme exemplars of the same categories (e.g. Hitler) leads to contrasted subsequent judgments. Stapel and his colleagues (Dijksterhuis, Spears, Postmes, Stapel, Koomen, van Knippenberg \& Scheepers, 1998; Stapel, Koomen, \& van der Pligt, 1997) argued that exemplars of a category constitute a comparison standard for evaluating new stimuli. If the primed exemplar is sufficiently extreme, this results in comparative contrast, which is not produced by participants' intentional correction attempts (correction contrast). Applied to the present study, it is conceivable that participants in the overt priming condition compared themselves with the 'I', that they described in the created sentences. This may have resulted in comparison contrast, rather than correction contrast. In particular, if the 'I' described in the unscrambled sentences appeared to be extremely independent (i.e. unique), one person's similarity to another seems comparatively large. If this 'I' appeared to be extremely interdependent (i.e. similar), however, self-other similarity should be perceived as comparatively small. Participants in the subtle priming condition did not write down sentences including 'I'. Therefore, the categories 'independence' and 'interdependence' were activated, but no extreme exemplar that could have served as a comparison standard for evaluating one's similarity with another person was primed. As a result, assimilation occurred in the subtle priming condition, but contrast in the overt condition.

This alternative interpretation of our results cannot be dismissed with the given data. To summarize, our results do not tell us if the contrast effect observed in the overt priming condition is due to correction contrast or to comparative contrast. Nevertheless, the contribution of our results for studying the influence of independent and interdependent self-knowledge is twofold. First, we have shown that priming independent and interdependent self-knowledge influences social comparisons, by using a method that differs from the ones employed in earlier studies. Second, the present study gives evidence that the direction of this influence depends on the subtlety of the priming. In the beginning we introduced the concept of independenceinterdependence by mentioning cross-cultural differences, which have been traced back to variations in the construal of identity. As we argued, the causal assumption underlying these studies can not be directly tested with quasi-experimental research designs typically used in cross-cultural comparisons. Further research testing the consequences of priming independent and interdependent self-knowledge is therefore required in order to substantiate the assumption that the self-construals are critical in determining our psychological experiences in various domains. 


\section{ACKNOWLEDGEMENTS}

The research reported here was supported by the Deutsche Forschungsgemeinschaft (DFG), grant HA 2381/3-1.

\section{REFERENCES}

Bargh JA, Chen M, Burrows L. 1996. Automaticity of social behavior: Direct effects of trait construct and stereotype activation on action. Journal of Personality and Social Psychology 71: $230-244$.

Brewer MB, Gardner W. 1996. Who is this 'we'? Levels of collective identity and self representations. Journal of Personality and Social Psychology 71: 83-93.

Dijksterhuis A, Spears R, Postmes T, Stapel D, Koomen W, van Knippenberg A, Scheepers D. 1998. Seeing one thing and doing another: Contrast effects in automatic behavior. Journal of Personality and Social Psychology 75: 862-871.

Gardner W, Gabriel S, Lee A. 1999. 'I' value freedom but 'we' value relationships: Selfconstrual priming mirrors cultural differences in judgment. Psychological Science.

Glaser J, Banaji MR. 1999. When fair is foul and foul is fair: Reverse priming in automatic evaluation. Journal of Personality and Social Psychology 77: 669-687.

Goethals GR, Messick DM, Allison ST. 1991. The uniqueness bias: studies of constructive social comparison. In Social Comparison: Contemporary theory and research, Suls J, Wills TA (eds). Erlbaum: Hillsdale, NJ; 149-176.

Hannover B. 1997. Das dynamische Selbst. Zur Kontextabhängigkeit selbstbezogenen Wissens. Huber: Bern, Switzerland [The dynamic self: The context-dependency of self-related knowledge.].

Herr PM. 1986. Consequences of priming: Judgment and behavior. Journal of Personality and Social Psychology 51: 1106-1115.

Higgins ET, King G. 1981. Accessibility of social constructs: Information processing consequences of individual and contextual variability. In Personality, Cognition, and Social Interaction, Cantor N, Kihlstrom JF (eds). Erlbaum: Hillsdale, NJ; 69-121.

Higgins ET, Rholes WS, Jones CR. 1977. Category accessibility and impression formation. Journal of Experimental Social Psychology 13: 141-154.

Kuhn MH, McPartland TS. 1954. An empirical investigation of self-attitudes. American Sociological Review 19: 68-76.

Kühnen U. 1999. Verzerrungen bei direkten und indirekten selbstbezogenen Ähnlichkeitsurteilen als Konsequenz hoch zugänglichen Selbstwissens. Pabst, Science Publishers: Lengerich, Germany [Biases in direct and indirect self-other similarity judgments as a consequence of highly accessible self-knowledge].

Markus HR, Kitayama S. 1991. Culture and the self: Implications for cognition, emotion, and motivation. Psychological Review 98: 224-253.

Markus HR, Kitayama S, Heiman RJ. 1997. Culture and 'basic' psychological principles. In Social Psychology: Handbook of basic principles, Higgins ET, Kruglanski AW (eds). Guilford: New York.

Miller JG, Bersoff DM, Harwood RL. 1990. Perception of social responsibilities in India and the United States: Moral imperatives or personal decisions? Journal of Personality and Social Psychology 58: 33-47.

Moskowitz GB, Roman RJ. 1992. Spontaneous trait inferences as self-generated primes: Implications for conscious social judgment. Journal of Personality and Social Psychology 62: 728-738.

Mussweiler T. 2000. Direction of Comparison as a Determinant of Assimilation Versus Contrast in Social Comparison. Northwestern University: Chicago, IL. 
Mussweiler T, Strack F. in press. Consequences of social comparison: selective accessibility, assimilation and contrast. In Handbook of Social Comparison: Theory and Research, Suls J, Weeler L (eds). Plenum: New York.

Niedenthal PM, Beike DR. 1997. Interrelated and isolated self-concepts. Personality and Social Psychology Review 1: 106-128.

Oyserman D, Markus HR. 1996. The self as social representation. In Psychology of the Social, Moscovici S, Flick U (eds). Rowohlt Taschenbuch Verlag: Berlin; 38-70.

Rhee E, Uleman J, Lee H, Roman R. 1995. Spontaneous self-descriptions and ethnic identities in individualist and collectivist cultures. Journal of Personality and Social Psychology 69(1): $142-152$.

Schwartz SH. 1992. Universals in the content and structure of values: Theoretical advances and empirical test in 20 countries. In Advances in Experimental Social Psychology, Zanna M (ed.). Academic Press: Orlando, FL.

Shweder RA, Bourne EJ. 1984. Does the concept of a person vary cross-culturally. In Culture Theory: Essays on mind, self, and emotion, Shweder RA, LeVine RA (eds). Cambridge University Press: Cambridge.

Singelis TM. 1994. The measurement of independent and interdependent self-construals. Personality and Social Psychology Bulletin 20: 580-591.

Srull TK, Wyer RS. 1979. The role of category accessibility in the interpretation of information about persons: Some determinants and implications. Journal of Personality and Social Psychology 37: 1660-1672.

Stapel DA, Koomen W, van der Pligt J. 1997. Categories of category accessibility: The impact of trait versus exemplar priming on person judgments. Journal of Experimental Social Psychology 33: 44-76.

Strack F. 1992. The different routes to social judgment: Experiential versus informational strategies. In The Construction of Social Judgment, Martin LL, Tesser A (eds). Erlbaum: Hillsdale, NJ; 249-275.

Strack F, Hannover B. 1996. Awareness of the influence as a precondition for implementing correctional goals. In The psychology of action: Linking cognition and motivation of behavior, Gollwitzer PM, Bargh JA (eds). Guilford: New York.

Strack F, Schwarz N, Bless H, Kübler A, Wänke M. 1993. Awareness of the influence as a determinant of assimilation versus contrast. European Journal of Social Psychology 23(1): $53-62$.

Trafimow D, Finlay KA. 1996. The importance of subjective norms for a minority of people: Between-subjects and within-subjects analyses. Personality and Social Psychology Bulletin 22(8): 820-828.

Trafimow D, Smith MD. 1998. An extension of the 'two-baskets' theory to Native Americans. European Journal of Social Psychology 28(6): 1015-1019.

Trafimow D, Silverman ES, Mei-Tai Fan R, Shui Fun Law J. 1997. The effects of language and priming on the relative accessibility of the private and the collective self. Journal of Crosscultural Psychology 28: 107-123.

Trafimow D, Triandis HC, Goto SG. 1991. Some tests of the distinction between the private self and the collective self. Journal of Personality and Social Psychology 60: 649-655.

Wegener DT, Petty RE. 1997. The flexible correction model: The role of naive theories of bias in bias correction. Advances in Experimental Social Psychology 29: 141-208.

Ybarra O, Trafimow D. 1998. How priming the private self or collective self affects the relative weights of attitudes and subjective norms. Personality and Social Psychology Bulletin 24(4): $362-370$. 